\title{
THE ORIGIN OF DUST IN THE EARLY UNIVERSE: PROBING THE STAR FORMATION HISTORY OF GALAXIES BY THEIR DUST CONTENT
}

\author{
Eli DWeK ${ }^{1}$ AND IsABelle CHERCHNEFF ${ }^{2}$ \\ ${ }^{1}$ Observational Cosmology Lab, Code 665, NASA Goddard Space Flight Center, Greenbelt, MD 20771, USA; eli.dwek@nasa.gov \\ 2 Department Physik, Universität Basel, CH-4056 Basel, Switzerland; isabelle.cherchneff@ unibas.ch \\ Received 2010 August 13; accepted 2010 November 3; published 2011 January 4
}

\begin{abstract}
Two distinct scenarios for the origin of the $\sim 4 \times 10^{8} M_{\odot}$ of dust observed in the high-redshift $(z=6.4)$ quasar $\mathrm{J} 1148+5251$ have been proposed. The first assumes that this galaxy is much younger than the age of the universe at that epoch so that only supernovae ( $\mathrm{SNe}$ ) could have produced this dust. The second scenario assumes a significantly older galactic age, so that the dust could have formed in lower-mass asymptotic giant branch (AGB) stars. Presenting new integral solutions for the chemical evolution of metals and dust in galaxies, we offer a critical evaluation of these two scenarios and observational consequences that can discriminate between the two. We show that AGB stars can produce the inferred mass of dust in this object, however, the final mass of surviving dust depends on the galaxy's star formation history (SFH). In general, SNe cannot produce the observed amount of dust unless the average SN event creates over $\sim 2 M_{\odot}$ of dust in its ejecta. However, special SFHs can be constructed in which SNe can produce the inferred dust mass with a reasonable average dust yield of $\sim 0.15 M_{\odot}$. The two scenarios propose different origins for the galaxy's spectral energy distribution, different star formation efficiencies and stellar masses, and consequently different comoving number densities of J1148+5251-type hyperluminous infrared (IR) objects. The detection of diagnostic mid-IR fine structure lines and more complete surveys determining the comoving number density of these objects can discriminate between the two scenarios.
\end{abstract}

Key words: galaxies: evolution - galaxies: high-redshift - galaxies: starburst - infrared: galaxies - quasars: individual (SDSS J114816.64+525150.3)

Online-only material: color figures

\section{INTRODUCTION}

Determining the origin of the massive amount of dust present in Sloan Digital Sky Survey (SDSS) J1148+5251 (hereafter $\mathrm{J} 1148+5251$ ), a hyperluminous quasar at $z=6.4$, presents a special challenge. The dust mass inferred from far-infrared and submillimeter observations is $\sim(1-5) \times 10^{8} M_{\odot}$ depending on dust composition (Dwek et al. 2007 and references therein), about 10 times larger than the mass of dust in the Milky Way (MW; Sodroski et al. 1997). The total far-infrared (IR) luminosity is about $2 \times 10^{13} L_{\odot}$, giving a star formation rate (SFR) of $\sim 3400 M_{\odot} \mathrm{yr}^{-1}$ for a Salpeter initial mass function (IMF; Kennicutt 1998a). Since the universe was only $\sim 890 \mathrm{Myr}$ old at that redshift, and the galaxy perhaps much younger, it has been suggested that only core-collapse supernovae (CCSNe) can produce the observed amount of dust in this object (Morgan \& Edmunds 2003; Dunne et al. 2003; Nozawa et al. 2003; Maiolino et al. 2004; Rho et al. 2008; Sibthorpe et al. 2010). Another argument made in favor of $\mathrm{SNe}$ as the most important dust sources in the early universe was based on observations of unmixed ejecta of young remnants, primarily Cas A. The inferred dust mass in this remnant, ranging from $\sim 0.1$ to $0.15 M_{\odot}$, was considered sufficiently large for SNe to account for the observed dust mass in J1148+5251 (Rho et al. 2008; Sibthorpe et al. 2010; Barlow et al. 2010). We consider the $\sim 1 M_{\odot}$ of dust mass claimed by Dunne et al. $(2003,2009)$ to have formed in Cas A as an unreasonable amount of dust. The total ejecta mass is $2-4 M_{\odot}$, of which only $\sim 0.2 M_{\odot}$ consists of condensible elements (e.g., Nozawa et al. 2010 and references therein).

None of these assertions, that $\mathrm{SNe}$ are significant dust sources in the early universe, were substantiated by detailed calculations. Specifically, their role as destroyers of dust during the remnant phase of their evolution was completely ignored. Furthermore, these claims tacitly assume that the epoch of intense star formation spanned the entire age of the galaxy, which requires an excessively large reservoir of interstellar gas. Finally, recent calculations show that only $\sim 0.15 M_{\odot}$ of dust is created in a Population III $20 M_{\odot}$ SN (Cherchneff \& Dwek 2010), which may be a typical yield for Pop II SNe of similar masses. All these considerations point to the need for more detailed calculations to ascertain the role of $\mathrm{SNe}$ as dust sources and sinks in the early universe. In such recent calculations, Dwek et al. (2007) explored the combined effects of dust formation and destruction on the net amount of dust produced in J1148+5251. They found that $\mathrm{SNe}$ cannot be important sources of dust in the early universe unless they produce significantly more dust and destroy significantly less dust than implied by current observations or theoretical calculations.

In light of these difficulties, Valiante et al. (2009) suggested that asymptotic giant branch (AGB) stars could be the source of dust in J1148+5251. Their model was motivated by the numerical simulations of the formation and growth of this galaxy through a series of successive mergers that resulted in repeated intense bursts of star formation ( $\mathrm{Li}$ et al. 2007, hereafter Li07). Associated with these mergers is the growth of its central black hole $(\mathrm{BH})$, which at $z \approx 6.4$ has reached a mass of about $\sim 10^{9} M_{\odot}$. In this scenario, star formation commenced at $z \approx 15$, when the universe was merely $250 \mathrm{Myr}$ old. Consequently, the progenitors of the more numerous and efficient dust producing AGB stars had time to evolve off the main sequence (MS; see Table 1) and produce the observed mass of dust in J1148+5251.

This paper takes a critical look at these two proposed dust formation scenarios, hereafter referred to as the SN and AGB scenarios. We start by introducing some basic definitions of 
Table 1

Main-sequence Lifetimes of AGB Progenitor Stars ${ }^{\mathrm{a}}$

\begin{tabular}{lrrrrr}
\hline \hline Mass & \multicolumn{5}{c}{ Metallicity } \\
\cline { 2 - 6 }$\left(M_{\odot}\right)$ & 0.0001 & 0.001 & 0.008 & 0.0100 & 0.02 \\
\hline 1.0 & 4998 & 6774 & 8634 & 8846 & 9516. \\
1.5 & 1474 & 2022 & 2415 & 2446 & 2528. \\
2.0 & 663 & 914 & 1047 & 1054 & 1062. \\
2.25 & 486 & 670 & 756 & 759 & 757. \\
2.5 & 371 & 512 & 570 & 570 & 565. \\
2.75 & 292 & 404 & 444 & 443 & 436. \\
3.0 & 236 & 326 & 355 & 354 & 346. \\
3.25 & 195 & 270 & 291 & 290 & 282. \\
3.5 & 164 & 227 & 243 & 241 & 234. \\
3.75 & 140 & 194 & 206 & 204 & 197. \\
4.0 & 121 & 168 & 177 & 175 & 168. \\
4.5 & 94 & 129 & 135 & 134 & 128. \\
5.0 & 75 & 104 & 107 & 106 & 100. \\
5.5 & 62 & 85 & 87 & 86 & 81. \\
6.0 & 52 & 72 & 73 & 72 & 68. \\
6.5 & 45 & 61 & 62 & 61 & 57. \\
7.0 & 39 & 53 & 53 & 53 & 49. \\
7.5 & 34 & 47 & 47 & 46 & 43. \\
8.0 & 31 & 42 & 41 & 41 & 38. \\
\hline & & & & & \\
\hline
\end{tabular}

Note. ${ }^{\text {a }}$ Lifetimes in Myr, calculated using the analytical formulae from Raiteri et al. (1996).

the various quantities that govern the chemical evolution of galaxies and present new integral solutions for the chemical evolution of their elemental and dust content (Section 2). In Section 3, we discuss the yields of the main stellar dust sources. These include the explosive SN ejecta, the fast winds created during the Wolf-Rayet (W-R) stage of the evolution of stars with masses in excess of $\sim 40 M_{\odot}$, and the quiescent winds from AGB stars. In Section 4, we apply the model to follow the chemical evolution of $\mathrm{J} 1148+5251$. We calculate the contribution of $\mathrm{SNe}$ and AGB stars to the production of dust in this galaxy and the evolution of its stellar mass and luminosity. The simulated star formation history (SFH) of Li07 leading to the formation of $\mathrm{J} 1148+5251$ is not unique, and in Section 5, we examine alternate scenarios for the formation and survival of dust by AGB stars and SNe. Our results show that both the AGB and the $\mathrm{SN}$ scenarios are still viable for producing the mass of dust observed in $\mathrm{J} 1148+5251$, each scenario with its own problems and limitations. We therefore present in Section 6 observational tests, including studies of the spectral energy distribution (SED), the rarity of J1148+5251-type objects, and the inferred SFR, that can discriminate between the two. In Section 7, we explore two additional, non-stellar, dust sources: molecular clouds (MCs), which have always been considered as an environment for the growth and processing of interstellar dust grains in the galaxy (Snow 1975; Dwek \& Scalo 1980; Liffman \& Clayton 1989; Dwek 1998; Greenberg \& Li 1999; Zhukovska et al. 2008) and active galactic nucleus (AGN) winds, which were recently suggested as potential producers of interstellar dust in quasars (Elvis et al. 2002; Maiolino et al. 2006). The results of the paper are briefly summarized in Section 8 .

Throughout this paper we adopt a flat $\Lambda$ CDM cosmology, with a baryonic density parameter $\Omega_{b}=0.044$, a total matter (dark+baryonic) density parameter of $\Omega_{m}=0.27$, a vacuum energy density $\Omega_{\Lambda}=0.73$, and a Hubble constant of $H_{0}=$ $70 \mathrm{~km} \mathrm{~s}^{-1} \mathrm{Mpc}^{-1}$ (Spergel et al. 2007).

\section{EQUATIONS FOR THE EVOLUTION OF DUST}

\subsection{Basic Definitions}

We define the stellar IMF, $\phi(m)$, so that $\phi(m) d m$ is the number of stars with masses between $m$ and $m+d m$ and normalize it to unity in the $\left\{m_{l}, m_{u}\right\}$ mass interval, where $m_{l}$ and $m_{u}$ are, respectively, the lower and upper mass limits of the IMF. The IMF-averaged stellar mass, $\langle m\rangle$, is then

$$
\langle m\rangle=\int_{m_{l}}^{m_{u}} m \phi(m) d m .
$$

The SFR, $\psi(t)$, is the mass of stars formed per unit time and is related to the stellar birthrate, $B(t)$, by

$$
B(t)=\frac{\psi(t)}{\langle m\rangle} .
$$

We assume that all stars in the $\left[m_{l}, m_{w}\right]$ mass range end their life quiescently, whereas all stars with masses $m_{w} \leqslant m \leqslant m_{u}$ become CCSNe, where $m_{w}=8 M_{\odot}$ is their lower mass cut.

A useful quantity is $m_{\star}$, the mass of all stars born per SN event, given by

$$
m_{\star} \equiv\langle m\rangle / \int_{m_{w}}^{m_{u}} \phi(m) d m .
$$

The $\mathrm{SN}$ rate, $R_{S N}$, is then given by

$$
R_{S N}(t)=B(t) \int_{m_{w}}^{m_{u}} \phi(m) d m=\frac{\psi(t)}{m_{\star}} .
$$

In all our calculations we will use a mass-heavy ISM characterized by a power law: $\phi(m) \sim m^{-\alpha}$ in the $\left\{m_{l}, m_{u}\right\}=$ $\left\{1 M_{\odot}, 100 M_{\odot}\right\}$ mass interval, with $\alpha=2.35$. For this IMF we get

$$
\langle m\rangle=3.1 M_{\odot} \quad \text { and } \quad m_{\star}=53.0 M_{\odot} .
$$

The choice of this mass-heavy IMF is motivated by studies suggesting that star formation in the early universe was biased toward more massive stars (Bromm \& Larson 2004). For comparison, a Salpeter IMF gives values of $\langle m\rangle \approx 0.35 M_{\odot}$ and $m_{\star} \approx 135 M_{\odot}$.

\subsection{Equations for the Evolution of Dust or Elements}

We first describe the equations for the evolution of the mass of dust in the ISM. Let $M_{g}(t)$ be the mass of the gas in the ISM at a given time $t$. The evolution of $M_{d}(t)$, the mass of dust in the ISM, is governed by the equation

$$
\frac{d M_{d}(t)}{d t}=-Z_{d}(t) \psi(t)-\frac{M_{d}(t)}{\tau_{d}(t)}+\mathcal{S}(t) \pm\left[\frac{d M_{d}(t)}{d t}\right]_{\mathrm{inf} / \mathrm{out}},
$$

where $Z_{d}(t)$ is the dust-to-gas mass ratio:

$$
Z_{d}(t) \equiv \frac{M_{d}(t)}{M_{g}(t)}
$$

The first term in Equation (6) represents the rate at which the dust is removed from the ISM by star formation; the second is the rate at which dust is destroyed by sputtering or grain-grain collisions in SN blast waves. The parameter $\tau_{d}(t)$ is the timescale for the combined effect of these processes; the third term, $\mathcal{S}(t)$, 
is a source function representing the rate of dust formation in the different astrophysical environments; and the fourth term represents the rate of increase/decrease in the dust mass as a result of infall/outflow from the galaxy.

The timescale for grain destruction in $\mathrm{SN}$ shock waves is given by (Dwek \& Scalo 1980)

$$
\tau_{d} \equiv \frac{M_{d}}{\left\langle m_{d}\right\rangle R_{S N}} .
$$

The parameter $\left\langle m_{d}\right\rangle$ is the total mass of the refractory elements, initially locked up in dust, which are returned back to the gas phase of the ISM throughout the evolution of a single supernova remnant (SNR). For the MW galaxy, Jones (2004) found dust lifetimes of 400 and $600 \mathrm{Myr}$ for silicate and carbon grains, respectively. We adopt here an average lifetime of $\tau_{d} \approx 500$ Myr. This average should be time dependent since the dust composition, specifically the relative abundance of silicate and carbon dust, evolves with time. However, such distinction is not warranted in the present investigation because of our current lack of knowledge of the nature of the dust in highredshift galaxies. Adopting a total dust mass $M_{d} \approx 3 \times 10^{7} M_{\odot}$ and a Galactic SN rate $R_{S N} \approx 0.02$ (Diehl et al. 2006) gives a value of about $3 M_{\odot}$ for the grain destruction efficiency in the Galaxy. Since $\left\langle m_{d}\right\rangle$ scales linearly with the mass of ISM dust, we can normalize it to its Galactic value:

$$
\left\langle m_{d}\right\rangle=\left[\frac{Z_{d}(t)}{Z_{d, m w}}\right]\left\langle m_{d}\right\rangle_{m w},
$$

where $\left\langle m_{d}\right\rangle_{m w}=3 M_{\odot}$ is the MW value of $\left\langle m_{d}\right\rangle$ and $Z_{d, m w} \approx$ 0.007 is the average dust-to-gas mass ratio in the MW (Zubko et al. 2004).

The grain destruction rate can then be written as

$$
\frac{M_{d}(t)}{\tau_{d}(t)}=Z_{d}(t)\left[\frac{\left\langle m_{d}\right\rangle_{m w} R_{S N}}{Z_{d, m w}}\right] .
$$

The source function $\mathcal{S}(\mathrm{t})$ represents the rate at which the dust mass in the ISM increases by nucleation in the different dust sources, or by growth in the ISM, which is an important process responsible for the differential depletions in its different phases. Considering only stellar sources, the source function can be written as

$$
\begin{aligned}
& \mathcal{S}(t)=\int_{\widetilde{m}(t)}^{m_{w}}\left[\frac{\psi\left(t-\tau_{m s}(m)\right)}{\langle m\rangle}\right] Y_{d, a g b}(m) \phi(m) d m \text { (AGB stars) } \\
& +\int_{m_{w r}}^{m_{u}}\left[\frac{\psi\left(t-\tau_{m s}(m)\right)}{\langle m\rangle}\right] Y_{d, w r}(m) \phi(m) d m \quad \text { (WR stars) } \\
& +\int_{m_{w}}^{m_{u}}\left[\frac{\psi\left(t-\tau_{m s}(m)\right)}{\langle m\rangle}\right] Y_{d, s n}(m) \phi(m) d m \quad(\mathrm{SNe}) \\
& +\mathcal{A}_{I a} \int_{0}^{t}\left[\frac{\psi(t-\tau)}{\langle m\rangle}\right] Y_{d, I a} f_{I a}(\tau) d \tau \quad(\mathrm{SNIa}),
\end{aligned}
$$

where the different terms represent the net contribution of the different stellar sources to the dust mass in the ISM. For each stellar mass, the SFR, $\psi\left(t^{\prime}\right)$, is calculated at the epoch $t^{\prime}=t-\tau_{m s}(m)$, where $\tau_{m s}(m)$ is the MS lifetime of a star of mass $m$ (Table 1). For AGB stars, the lower limit of the integral, $\tilde{m}(t)$, is given by $\max \left\{m_{l}, m^{\prime}(t)\right\}$, where $m^{\prime}(t)$ is the mass of a star with a MS lifetime $t$. The lower limit, $m_{w r}$, is the limiting mass above which stars undergo extensive mass loss and become
WR stars. $Y_{d, a g b}(m), Y_{d, w r}(m), Y_{d, s n}(m)$, and $Y_{d, I a}$ are the dust yields in the quiescent winds of AGB and WR stars, and in the explosive ejecta of core-collapse and Type Ia SNe, respectively. In the last term, $\mathcal{A}_{I a}$ is a normalization constant that can be determined from the ratio between the observed frequency of Type II and Type Ia SNe, and $f_{I a}(\tau)$ is the distribution function of the delay time, $\tau$, between the birth of the stellar system and the SNIa event (Greggio 2005, 2010).

Finally, the yields in Equation (11), except the SNIa yields, depend on the initial stellar metallicity. This implicit time dependence is suppressed in the equations and its implementation in the solution is described in Section 2.4 below.

For stellar masses above $\sim 8 M_{\odot}, \tau_{m s}(m) \ll t$ for most times of interest, so that the yield from $\mathrm{SNe}$ and WR stars can be set equal to $R_{S N} \widetilde{Y}_{d, s n}$, and $R_{W R} \widetilde{Y}_{d, w r}$, respectively. $R_{W R}$ is the rate of WR stars, which can be defined similarly to the SN rate (see Equation (4)) with $m_{w}$ replaced by $m_{w r}$. The IMF-averaged yields of SNe and WR stars are given by

$$
\tilde{Y}_{d, X}=\int_{m_{X}}^{m_{u}} Y_{d, X}(m) \phi(m) d m / \int_{m_{X}}^{m_{u}} \phi(m) d m,
$$

where $X \equiv s n$ or $w r$.

The term describing the rate of change in the dust mass caused by the exchange of gas with the intergalactic medium depends on whether the gas is flowing into or out of the galaxy. In an outflow, the dust-to-gas mass ratio in the outflowing gas is about equal to the galaxy's value, $Z_{d}(t)$, and the term is given by

$$
\left[\frac{d M_{d}(t)}{d t}\right]_{\text {out }}=Z_{d}(t)\left(\frac{d M_{g}(t)}{d t}\right)_{\text {out }} \quad \text { for outflows. }
$$

In the case of infall, the dust-to-gas mass ratio of the infalling gas, $Z_{d}^{\mathrm{inf}}(t)$, is an independent variable and the term becomes

$$
\left[\frac{d M_{d}(t)}{d t}\right]_{\mathrm{inf}}=Z_{d}^{\mathrm{inf}}(t)\left(\frac{d M_{g}(t)}{d t}\right)_{\mathrm{inf}} \quad \text { for infalls. }
$$

\subsection{The Evolution of the Gas}

The evolution of the ISM gas is given by the equation

$$
\begin{aligned}
\frac{d M_{g}(t)}{d t}= & -\psi(t)+\int_{m_{l}}^{m_{u}}\left[\frac{\psi\left(t-\tau_{m s}(m)\right)}{\langle m\rangle}\right] M_{e j}(m) \phi(m) d m \\
& \pm\left[\frac{d M_{g}(t)}{d t}\right]_{\text {inf/out }} \\
\approx & -\left(1-R_{e j}\right) \psi(t) \pm\left[\frac{d M_{g}(t)}{d t}\right]_{\text {inf/out }},
\end{aligned}
$$

where the first term in the top line represents the conversion rate of the ISM mass into stars, the second term represents the rate of stellar mass loss, where $M_{e j}(m)$ is the total mass returned back to the ISM by a star of initial mass $m$, and the third term represents the rate of change in the ISM mass as a result of gas infall or outflow. The second line represents the behavior of $d M_{g}(t) / d t$ in the instantaneous recycling approximation, where the returned (ejected) mass fraction, $R_{e j}$, is approximately 0.7 .

The evolution of the gas mass is only weakly coupled to its chemical evolution. The returned mass, $M_{e j}(t)$, is almost independent of metallicity (Karakas \& Lattanzio 2007) and the stellar MS lifetimes are only weakly dependent on metallicity for values above 0.001. So, with reasonable accuracy, the evolution of the gas mass can (but does not need to) be solved independently of the evolution of the dust and metals in the ISM. 


\subsection{Integral Solutions}

When the evolution of the ISM gas is decoupled from its chemical evolution, Equation (6) can be readily solved with the aid of an integration factor to yield a convenient functional form for the evolution of the dust or any element in the ISM.

Changing variables (Audouze \& Tinsley 1976)

$$
\frac{d M_{d}(t)}{d t}=M_{g}(t) \frac{d Z_{d}(t)}{d t}+Z_{d}(t) \frac{d M_{g}(t)}{d t},
$$

Equation (6) can be rewritten as

$$
\frac{d Z_{d}(t)}{d t}+Z_{d}(t) F(t)=G(t)
$$

where $F(t)$ and $G(t)$ are dimensional functions (units of time ${ }^{-1}$ ) given by

$$
\begin{aligned}
F(t) \equiv & \frac{\psi(t)}{M_{g}(t)}\left[1+\frac{\left\langle m_{d}\right\rangle_{m w}}{m_{\star} Z_{d, m w}}+\frac{1}{\psi(t)} \frac{d M_{g}(t)}{d t}\right. \\
& \left.+\frac{1}{\psi(t)}\left(\frac{d M_{g}(t)}{d t}\right)_{\text {out }}\right]
\end{aligned}
$$

and

$$
G(t) \equiv \frac{1}{M_{g}(t)}\left[\mathcal{S}(t)+Z_{d}^{\mathrm{inf}}(t)\left(\frac{d M_{g}(t)}{d t}\right)_{\mathrm{inf}}\right]
$$

Note that the outflow term is included in $F(t)$ because it is proportional to $Z_{d}(t)$, whereas the infall term is included in the expression for $G(t)$, since it is independent of the evolving metallicity of the galaxy.

Equation (17) can be solved with the aid of an integration factor $\lambda(t)$, which is defined as

$$
\lambda(t)=\exp \left[\int F(t) d t\right]
$$

giving

$$
Z_{d}(t)=\lambda(t)^{-1} \int_{0}^{t} \lambda\left(t^{\prime}\right) G\left(t^{\prime}\right) d t^{\prime}+\mathcal{C},
$$

where $\mathcal{C}$ is an integration constant.

Given $M_{g}(t)$, the mass of dust is simply given by

$$
M_{d}(t)=Z_{d}(t) M_{g}(t)
$$

Finally, Equation (6) can also be used to describe the evolution of the metallicity, $Z_{A}$, of any stable or unstable element $A$ by the formal substitution of $M_{d}$ with $M_{A}$ and by using the appropriate yields in Equation (11). For stable elements, the parameter $\left\langle m_{d}\right\rangle_{m w}$ in Equation (18) should be set to zero and for an unstable element, the dust lifetime, $\tau_{d}$, in Equation (6) must be substituted by its radioactive decay time.

The solutions, as written here, are easily calculated for yields at a fixed metallicity, when the source function $S(t)$ has a simple time dependency. Taking the changes of $S(t)$ with metallicity into account can be achieved in a straightforward way. Yields are usually calculated for a finite grid of metallicities $\left\{Z_{0}(j), j=1, N\right\}$. One can therefore calculate the times $\left\{t_{j}, j=1, N\right\}$ when the solutions derived with stellar yields at the fixed metallicities, $M\left[Z_{0}(j), t\right]$, reach the corresponding metallicities $\left\{Z_{0}(j), j=1, N\right\}$. The final evolution of the mass, $M(t)$, of the elements or the dust can then be written as a sum

$$
M(t)=\sum_{1}^{N} w_{j}(t) M\left[Z_{0}(j), t\right],
$$

where $\left\{w_{j}(t), j=1, N\right\}$ are weight functions chosen to ensure the continuity of $M(t)$ across the grid of times $\left\{t_{j}\right\}$.

\section{STELLAR SOURCES OF INTERSTELLAR DUST}

In the following we examine the yields and relative importance of the dust formation in SNe, WR, and AGB stars. In all our calculations we adopt the previously described mass-heavy IMF.

\subsection{Supernovae}

Consider a constant $\mathrm{SN}$ rate, $R_{S N}$. The dust production rate is simply given by $S_{0}=\widetilde{Y}_{d, s n} \times R_{S N}$. Without grain destruction and with an SN rate of $20 \mathrm{yr}^{-1}$, SNe need to condense only $0.03 M_{\odot}$ of dust over a period of $\sim 500 \mathrm{Myr}$ in order to produce an observed dust mass of $\sim 3 \times 10^{8} M_{\odot}$. When grain destruction by $\mathrm{SN}$ is taken into account, the amount of dust produced by SNe is given by $M_{d} \approx \widetilde{Y}_{d, s n} R_{S N} \tau_{d}$, giving the trivial solution: $\widetilde{Y}_{d, s n} \approx\left\langle m_{d}\right\rangle$, that is, in a steady state SNe must produce a dust mass that is equal to the amount they destroy during their lifetime. However, the required value of $\widetilde{Y}_{d, s n}$ can be lower before the system reaches a steady state. For example, the first $\mathrm{SNe}$ expand in a dust-free medium and therefore are net producers of interstellar dust. Detailed chemical evolution and population synthesis models are therefore needed to fit the observational constraints imposed by the inferred stellar, gas, and dust masses, as well as by the stellar and radiative output of the galaxy.

Simple analytical models have shown that even in the absence of grain destruction, $\mathrm{SNe}$ must produce at least $\sim 0.3 M_{\odot}$ of dust to account for the observed dust-to-gas mass ratio in J1148+5251 (Dwek et al. 2007; their Figure 8). The reason that this number is not lower and equal to the value of $\sim 0.03 M_{\odot}$ derived in our simple estimate above stems from the fact that in these models the SFR is proportional to $\Sigma_{\text {gas }}^{1.5}$, where $\Sigma_{\text {gas }}$ is the mass surface density of the gas (Kennicutt 1998b). $\Sigma_{\text {gas }}$ evolves with time, so that the effective timespan over which star formation takes place is therefore much shorter than the age of the galaxy. It is determined by either the buildup of the ISM in infall models or by the depletion of the ISM in closed box models. For J1148+5251, the situation is exacerbated by the fact that the IR-inferred SFR is $\sim 3000 M_{\odot} \mathrm{yr}^{-1}$. This SFR can therefore only be sustained for about $20 \mathrm{Myr}$, if the reservoir of gas is comparable to the dynamical mass or the $\mathrm{CO}$ mass of the galaxy.

Do SNe actually produce this much dust? The most detailed information on the amount of dust formed in SNe comes from IR observations of the young SNR of Cas A. Spitzer observations of the remnant have revealed the presence of $\sim 0.02-0.04 M_{\odot}$ of hot dust (Rho et al. 2008) and $\sim 0.08 M_{\odot}$ of cool dust (Sibthorpe et al. 2010; Barlow et al. 2010). Furthermore, detailed calculations, using a chemical kinetic approach to follow the transformation of gas phase molecules into small clusters of dust precursors, show that only $\sim 0.1-0.15 M_{\odot}$ of dust is created in the explosive ejecta of a Population III $20 M_{\odot}$ SN (Cherchneff $\&$ Dwek 2010). Such yields may be typical of SNe of similar 

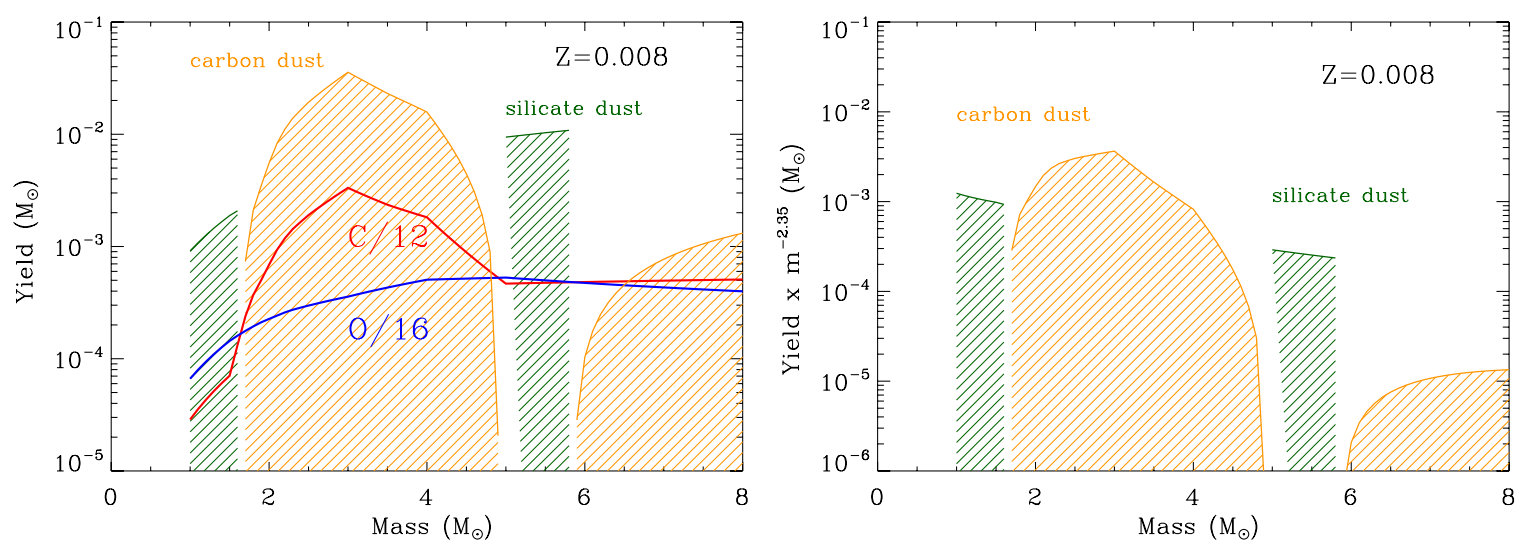

Figure 1. Left panel: the dust yield from AGB stars with an initial metallicity of 0.008 calculated using the yields of Karakas \& Lattanzio (2007). The red and blue lines depict the carbon and oxygen yields from these stars, divided by their atomic number. Carbon dust (hatched orange regions) is formed when the $\mathrm{C} / \mathrm{O}$ ratio $>1$, whereas silicate dust (hatched green region) is formed when that ratio is $<1$. Right panel: the IMF-weighted yield of dust from these stars. The $\sim 3 M_{\odot}$ stars, which have MS lifetimes of $\sim 350 \mathrm{Myr}$ (see Table 1) are the dominant contributors to the production of dust.

(A color version of this figure is available in the online journal.)

masses, regardless of the initial metallicity of the progenitor star. This yield is still not sufficient to account for the mass of dust observed in J1148+5251 except when grain destruction is almost nonexistent in that galaxy. Such special scenario is discussed in Section 4.1.

\subsection{Wolf-Rayet Stars}

Of the stars that become CCSNe, only those with masses above $m_{w r} \approx 40 M_{\odot}$ will become WR stars, a stage during which they will experience extensive mass loss. The formation of carbon dust becomes feasible during the latest phases of this mass loss phase, when the stellar surface becomes carbon enriched, and the star evolves into a carbon-rich WR star (WC star; Crowther 1997). For an IMF with a Salpeter slope of $\alpha=2.35$, the fraction of CCSNe that become WR stars is

$$
f_{w r} \approx\left(m_{w r} / m_{w}\right)^{1-\alpha} \approx 0.11 .
$$

So, WR stars have to produce about 10 times more dust than $\mathrm{SNe}$ to account for the dust mass in $\mathrm{J} 1148+5251$. There is observational evidence that most WR stars are part of a binary system with an $\mathrm{OB}$ companion and that the dust formation locus is located in the region where the two winds collide as exemplified by the archetypical systems WR104 and WR140. For the Pinwheel Nebula WR 104, a dust mass loss of $\sim 8 \times 10^{-7} M_{\odot} \mathrm{yr}^{-1}$ is derived by Harries et al. (2004). A typical $60 M_{\odot}$ WR star is characterized by a WC phase lasting about $2 \times 10^{5} \mathrm{yr}$ (Prantzos et al. 1986). We can thus approximate the amount of dust formed by WR stars as $\sim 0.2 M_{\odot}$ over their lifetime. This number is of the same order of magnitude and not 10 times larger than the dust mass formed in the ejecta of a CCSN. Therefore, WR stars are certainly contributing to the dust budget of J1148+5251 but no more than $10 \%$ of all dust makers.

\subsection{AGB Stars}

In the MW, a significant fraction of dust is produced in AGB stars (Dwek 1998; Tielens 1998). Depending on their evolutionary stage on the AGB, red giants have either an oxygenrich stellar photosphere characterized by a $\mathrm{C} / \mathrm{O}$ ratio $<1$ or a carbon-rich photosphere resulting from thermal pulses and third dredge-up and characterized by a C/O ratio $>1$. The former stars form silicate and metal oxide dust whereas the latter essentially form carbon dust and silicon carbides. At present, there exist no satisfactory models explaining the chemical formation of dust in AGBs and the impact of dust on accelerating the outflow through radiative pressure. Dynamical models often use the classical nucleation theory to describe dust synthesis and derive dust yields (Ferrarotti \& Gail 2006), an approach that has been proven to be inadequate when applied to circumstellar outflows (Donn \& Nuth 1985; Cherchneff \& Dwek 2010).

For the purpose of the present study, we ignore the chemical and physical complexity of the dust formation processes and assume a condensation efficiency of unity to calculate the dust yields as described by Dwek (1998). The calculations assume that carbon dust forms when the $\mathrm{C} / \mathrm{O}$ number ratio exceeds 1 and that silicate dust forms when this ratio is less than unity. This approach provides upper limits for the dust mass. For illustrative purposes, Figure 1 (left panel) depicts the mass of dust produced during the AGB phase from 1 to $8 M_{\odot}$ stars with an initial metallicity of 0.008 . Yields were taken from Karakas \& Lattanzio (2007). Also shown in the figure are the carbon and oxygen yields (in units of $M_{\odot}$ and divided by their atomic mass) to illustrate the range of stellar masses that produce either silicate or carbon dust. Yields similar to those presented in this figure were adopted by Valiante et al. (2009) based on the models of Ferrarotti \& Gail (2006). These models give different dust compositions, a distinction which is not important for the purpose of this paper.

The right panel of the figure shows the IMF-weighted yield of dust from these stars, where the IMF is characterized by a power law in mass with a Salpeter index of 2.35. The figure shows that the most efficient dust producers are $\sim 3 M_{\odot}$ stars, which have an MS lifetime of about $350 \mathrm{Myr}$. For AGB stars to be significant contributors to the reservoir of dust in a galaxy, their ages have to be significantly older than $\sim 400 \mathrm{yr}$.

\subsection{Relative Importance}

The rate of dust production by SNe, WR stars, and AGB stars is given by $\psi \times \widetilde{Y}_{d, s n} / m_{\star}, 0.11 \times \psi \times \widetilde{Y}_{d, s n} / m_{\star}$, and $\psi \times$ $\widetilde{Y}_{d, a g b} /\langle m\rangle$, respectively. Taking $0.15,0.2$, and $0.04 M_{\odot}$ as the respective yields of dust in these sources, we find that in a steady state their relative dust production rate is 1.0:0.11:4.6. AGB stars are therefore the most important sources of interstellar dust. However, this steady state is not immediately realized because of the delayed injection of AGB-condensed dust into the ISM. In 
the local group of galaxies, this delayed injection is manifested in the observed trend of the abundance of polycyclic aromatic hydrocarbons (PAHs) with galactic metallicity, which is taken as a proxy for galactic age (Dwek 2005; Galliano et al. 2008). The trend shows the existence of a threshold metallicity below which the abundance of PAH is very low. PAHs condense in the atmospheres of AGB stars and therefore represent AGBcondensed dust. So, at early times, the mass of dust in galaxies is dominated by SN-condensed dust. The dust evolution models presented in Galliano et al. (2008) suggest that the steady state when AGB sources are the dominant dust producers may only be reached after about $\sim 1-2$ Gyr of stellar evolution. PAHs can also be produced by shock processing of AGB-condensed carbon dust. However, both mechanisms, stellar condensation and shocks, need AGB stars as the source of dust, so the PAHmetallicity trend manifests the delayed injection of dust by AGB stars, even if most of the PAHs are formed in interstellar shocks.

\section{THE QUASAR J1148+5251}

\subsection{The Evolution of the ISM Gas and Dust Masses}

In the following, we will apply our new mathematical formalism to calculate the evolution of the gas and dust in $\mathrm{J} 1148+5251$. For AGB stars to be an important source of interstellar dust, star formation must have commenced at least 400 Myr before the epoch of observations, which is about 900 Myr. In their paper, Valiante et al. (2009) adopted the SFH derived by Li07 from simulations of hierarchical galaxy mergers taking place at redshifts $z \gtrsim 6.5$. The merger history of Li07 is not unique and just one possible scenario leading to the formation of a supermassive $\mathrm{BH}$ at $z \approx 6$. We use it for illustrative purposes only, since it fulfills the necessary requirement for AGB stars to be significant sources of dust at high redshift. Their SFH, taken from their Figure 7 in Li07, is reproduced as a bold solid line in the left panel of Figure 5.

An important ingredient of chemical evolution models is the evolution of the gas mass. We adopted two different prescriptions for calculating this quantity. In the first, we adopted a closed box model (no infall or outflow) with an initial gas mass of $8 \times 10^{11} M_{\odot}$ and used Equation (15) and the Li07 SFR to calculate its evolution. A value of $R_{e j}=0.48$ was chosen so that the stellar mass derived from the chemical evolution model would match that derived from the population synthesis model PÉGASE (see Section 4.2). In the second approach, we assumed that the SFR derived from the galaxy simulation follows the Schmidt-Kennicutt law in which $\psi(t)=A M_{\mathrm{g}}^{1.5}(t)$, where $A$ is a proportionality constant and a measure of the star formation efficiency in the galaxy. The value of $A$ was chosen to reproduce the model's SFR of $60 M_{\odot} \mathrm{yr}^{-1}$ at $t=900 \mathrm{Myr}$ with a gas mass of $\sim 5 \times 10^{10} M_{\odot}$.

In the calculations, we adopted an IMF-averaged dust yield of $\widetilde{Y}_{d, s n}=0.15 M_{\odot}$, the yield derived by Cherchneff \& Dwek (2010) for a $20 M_{\odot}$ primordial CCSNe. The yield of dust in AGB stars was calculated as in Dwek (1998) using the stellar elemental yields of Karakas \& Lattanzio (2007). The total dust yield from AGB stars is only weakly dependent on metallicity, so for simplicity we adopted AGB yields at a constant metallicity of $Z=0.008$. The stellar IMF was chosen to be a power law: $\phi(m) \sim m^{-\alpha}$ between 1 and $100 M_{\odot}$, with a spectral index of $\alpha=2.35$.

Figure 2 depicts the evolution of dust and gas masses for the two adopted evolutionary models for $M_{g}(t)$ : the closed box model (left panel) and the model described by the
Schmidt-Kennicutt SF law (right panel). The vertical line at $t=900 \mathrm{Myr}$ is the observed dust abundance, the length of the bar reflecting the range of dust masses for the different dust compositions (Dwek et al. 2007). The results of our calculations are in good agreement with those of Valiante et al. (2009). Not surprising, the detailed evolution differs from theirs, since it depends on their adopted evolutionary history of the gas mass, the grain destruction efficiency, and AGB yields, all quantities that differ somewhat from the ones used in this work. However, the main results, the final masses produced by SN- and AGBcondensed dust, are very similar. The figures show that AGB stars can readily produce the observed amount of dust with the proposed SFR and that SNe contribute less than $20 \%$ of the total dust mass at $t=900 \mathrm{Myr}$. They can therefore only be important dust sources if their IMF-averaged yield is $\sim 1 M_{\odot}$, confirming the results of the simpler analytical model of Dwek et al. (2007).

Figure 2 also shows that for the Schmidt-Kennicutt model there exist two epochs, occurring $\sim 100$ and $\sim 400$ Myr after the commencement of SF at $z \approx 15$, during which $\mathrm{SNe}$ produce over $\sim 10^{8} M_{\odot}$ of dust. It therefore seems that in an SF scenario consisting of either an extremely young or an intermediate-age burst of stars, SNe can produce the observed amount of dust, even with an average dust yield of $\sim 0.15 M_{\odot}$, without resorting to the need for AGB stars.

At the time of the intermediate burst, the SFR is over $10^{4} M_{\odot} \mathrm{yr}^{-1}$. Compared to the younger burst, this burst requires a significantly larger SFR to produce a similar amount of dust, a consequence of the effect of grain destruction. The corresponding bolometric luminosity is over $2 \times 10^{14} L_{\odot}$. Both the SFR and the luminosity are in excess of the observational constraints, ruling out this $\mathrm{SN}$ scenario as a viable source of dust.

The extremely young burst will produce a total bolometric luminosity of $\sim 5 \times 10^{13} L_{\odot}$, similar to the observed luminosity of $\sim 10^{14} L_{\odot}$, and a stellar mass of $\sim 10^{11} M_{\odot}$ (see Figure 3 ). This is a viable scenario for the formation of dust in J1148+5251, although it requires the formation of a supermassive $\mathrm{BH}$ on a very short timescale, and has implications on the comoving number and luminosity densities of such objects in the early universe (see Section 6.2 below).

\subsection{The Evolution the Stellar Luminosity and Mass}

Figure 3 depicts the evolution of the stellar masses and remnants (left panel) and the bolometric stellar luminosity (right panel) of J1148+5251. Calculations were done for the hierarchical SF history depicted in Figure 5, using the PÉGASE population synthesis code (Fioc \& Rocca-Volmerange 1997). Similar figures with identical results were presented by Li07 (Figures 11-12 in their paper). The merger history was proposed by them to account for the presence of a supermassive $\mathrm{BH}$ of mass $\sim 3 \times$ $10^{9} M_{\odot}$ (Willott et al. 2003). Assuming that the relationship between the BH mass and its spheroidal component is still valid at redshifts $\gtrsim 6$ gives a stellar bulge mass of $\sim$ few $\times 10^{12} M_{\odot}$ (e.g., review by Kormendy \& Gebhardt (2001)). While the stellar mass derived here agrees with that estimate, it is in excess of the dynamical mass of $\approx(5.0 \pm 2.5) \times 10^{10} M_{\odot}$ that is enclosed within a $2.5 \mathrm{kpc}$ radius of $\mathrm{J} 1148+5251$ (Walter et al. 2004). Walter et al. (2004) proposed several solutions to this discrepancy, including an overestimate in the mass of the $\mathrm{BH}$, and a breakdown in the assumption that the $\mathrm{CO}$ gas is gravitationally bound. Also, there may be evidence that at high redshifts, BHs may grow very rapidly without the corresponding increase in the mass of the host galaxy (Shields et al. 2006). It is therefore 

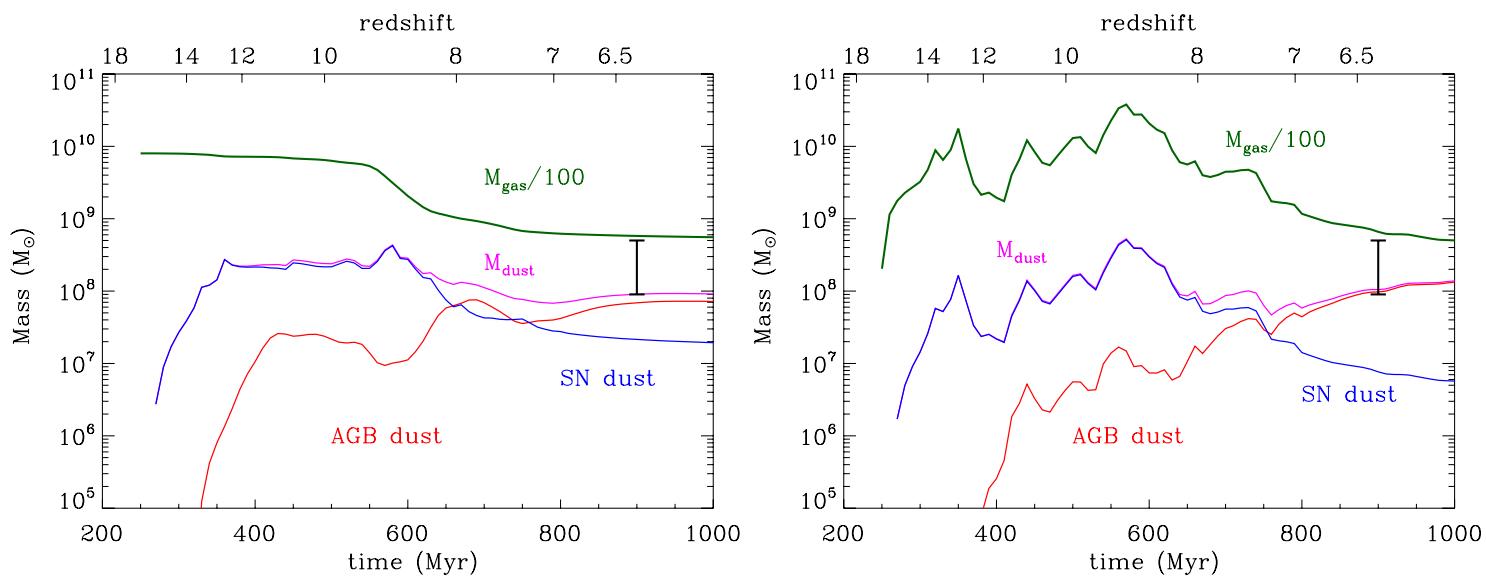

Figure 2. Evolution of the mass of the SN-condensed dust, AGB dust, total dust mass, and the mass of the ISM gas in J1148+5251, for the SF scenario of Li07, depicted here in Figure (5). Left panel: results for the closed box model. Right panel: results for the Schmidt-Kennicutt SF law. The vertical line at $t=900 \mathrm{Myr}$ is the observationally-inferred dust mass.

(A color version of this figure is available in the online journal.)
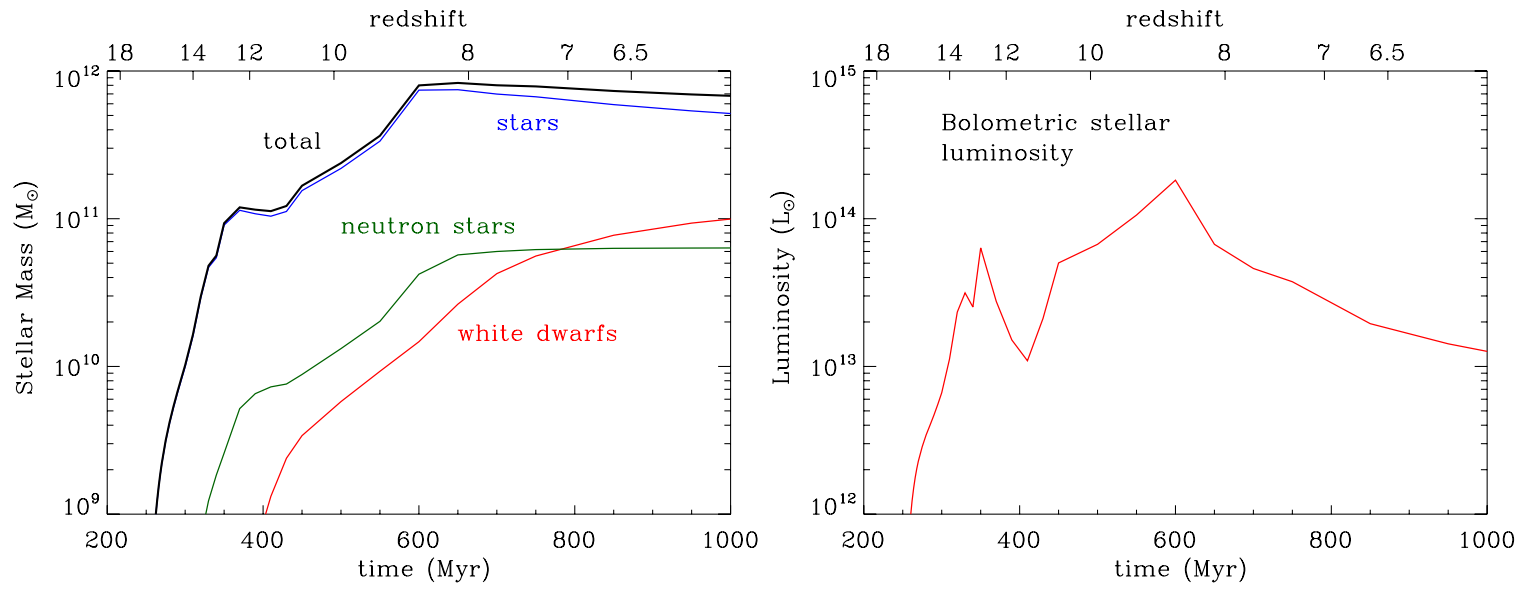

Figure 3. Evolution of the stellar mass content (left panel) and luminosity (right panel) calculated with the population synthesis code PÉGASE using the hierarchical SFH depicted in Figure 5.

(A color version of this figure is available in the online journal.)

premature to rule out the AGB dust formation scenario while the origin of this discrepancy is still unresolved.

\section{THE DEPENDENCE OF DUST MASS ON THE STAR FORMATION HISTORY}

As pointed out earlier, the Li07 SF scenario for the formation of $\mathrm{J} 1148+5251$ is not unique. We therefore explore the dependence of the total dust mass on the SFH of the galaxy. We first consider the production and survival of dust by single short bursts of star formation. Figure 4 depicts the evolution of the mass of dust produced by AGB stars that were born at time $t=0$ in a $100 \mathrm{Myr}$ burst of star formation characterized by a constant SFR of $1000 M_{\odot} \mathrm{yr}^{-1}$. The bold solid line depicts the net production of dust by AGB stars when no grain destruction occurs. It takes about $\sim 50 \mathrm{Myr}$ for the first AGB stars to evolve off the MS. The thinner lines depict the dust evolution in the presence of grain destruction. Grain destruction was assumed to be constant and to commence only after the cessation of the burst. After $600 \mathrm{Myr}$ the burst releases $\sim 1 \times 10^{8} M_{\odot}$ of dust if no destruction has taken place in the intervening period since the end of the burst and the time of observation. The surviving mass of dust is significantly smaller when grain destruction is taken into account. A young burst produces after $200 \mathrm{Myr}$ the least amount of dust, about $10^{7} M_{\odot}$ with no grain destruction, but is also least affected by grain destruction. The figure shows that a single burst of star formation (with no subsequent grain destruction), for example burst 1 with an intensity of $\sim 3000 M_{\odot}$ $\mathrm{yr}^{-1}$ or burst 4 with an intensity of $10,000 \mathrm{Myr}$, can produce the observed $\sim 3 \times 10^{8} M_{\odot}$ of dust in J1148+5251.

Deconstructing the complex SFH of a galaxy undergoing a series of hierarchical mergers can be very illustrative for understanding the origin of dust in such systems. In Figure 5 (left panel), we approximate the SFH of J1148+5251 used by Valiante et al. (2009) by five discrete bursts of star formation. The right panel depicts the cumulative contribution of each of the bursts to the total mass of dust in the galaxy. The oldest burst (number 1) releases the most amount of dust since all stars above $2.1 M_{\odot}$ contributed to its production. However, grain destruction by the subsequent bursts reduces the mass of dust that survives until $900 \mathrm{Myr}$ to only $\sim 2 \times 10^{6} M_{\odot}$. Almost all of the surviving dust was produced by the third burst. It has an MS turnoff mass of $\sim 3 M_{\odot}$, but also the largest SFR, and it is followed by a period of relatively low star formation activity and grain destruction.

The formation of about $\sim 3 \times 10^{8} M_{\odot}$ of dust at $z \gtrsim 6$ can therefore be achieved for different SFHs. As illustrated in Figure 4 , a single burst of star formation with the appropriate 


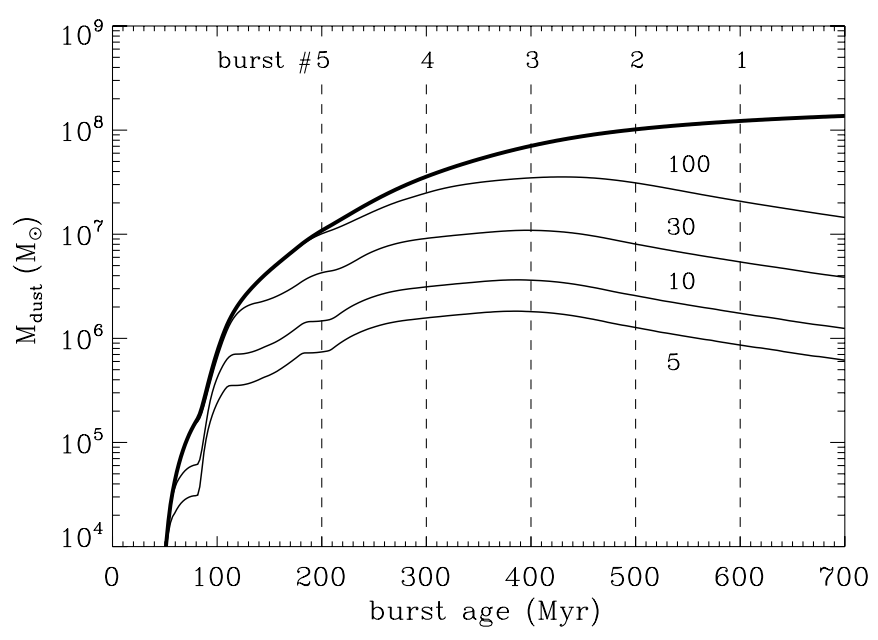

Figure 4. Evolution of the mass of dust released by AGB stars in a burst of star formation that commenced at $t=0$ as a function of time. The burst duration is $100 \mathrm{Myr}$, and its intensity is $1000 \mathrm{M}_{\odot} \mathrm{yr}^{-1}$. The top bold line represents the evolution of the dust mass without any grain destruction. Other lines depict the evolution of the dust when it is destroyed by a constant ongoing destruction mechanism, assumed to have started after the end of the burst. The lines are marked by the dust lifetime in units of Myr. The vertical dashed lines mark the age of the burst at epochs that correspond to the burst ages in Figure 5.

intensity can produce the required amount of dust, since no grain destruction takes place after the cessation of all star formation activities in the galaxy. Massive amounts of dust can also be produced by complex SFHs such as that presented in Figure 5 provided that the epochs of intense starburst activity are followed by a period with a very low rate, $\lesssim 70 M_{\odot} \mathrm{yr}^{-1}$, of star formation, so that the dust lifetime will be over $100 \mathrm{Myr}$. The discriminating factor between the different star formation histories capable of producing large amount of dust are the cumulative products, such as the metals or stellar masses, resulting from the star formation activities.

\section{OBSERVATIONAL DISCRIMINATION BETWEEN THE SN AND AGB SCENARIOS}

We have shown that not only AGB stars but also SNe can produce the inferred dust mass in $\mathrm{J} 1148+5251$, even with observed SN dust yields inferred from observations of Cas A. The SN scenario requires a special SF event, characterized by an intense burst of star formation that commenced just prior to the observations. The short duration of the burst can ensure that grain destruction did not have any time to significantly erode the newly formed dust. This is clearly an idealized scenario, since some grain destruction will take place when the reverse shock travels through the SN ejecta (Nozawa et al. 2010). In the following, we discuss several observational effects that can discriminate between the two scenarios.

\subsection{The Spectral Energy Distribution}

An important distinction between the SN and AGB scenarios for the origin of dust in $\mathrm{J} 1148+5251$ is the origin of its SED. In the SN scenario, most of the UV-optical and all the far-IR emission originate from starlight and starlight-heated dust. In the AGB scenario, the situation is more complicated and the origin of the SED depends on the fractions of the AGN and starburst luminosities that are absorbed and re-emitted by the dust. The total calculated stellar luminosity at $z=6.4(t \approx 900 \mathrm{Myr})$ is $1.6 \times 10^{13} L_{\odot}$ (see Figure 3 and Table 2$)$, about equal to the total far-infrared (FIR) luminosity, which is $\sim(2.0 \pm 0.5) \times 10^{13} L_{\odot}$.
Table 2

Energy Output from J1148+5251

\begin{tabular}{lc}
\hline \hline \multicolumn{1}{c}{ Wavelengths } & Luminosity $\left(L_{\odot}\right)$ \\
\hline UV-near-IR $(0.1-5 \mu \mathrm{m})$ & $(5.7 \pm 1.3) \times 10^{13}$ \\
Mid-IR $(5-30 \mu \mathrm{m})$ & $(1.8 \pm 0.2) \times 10^{13}$ \\
Far-IR $(30-300 \mu \mathrm{m})$ & $(2.5 \pm 0.5) \times 10^{13}$ \\
Bol $(0.1-300 \mu \mathrm{m})$ & $(10.0 \pm 1.5) \times 10^{13}$ \\
\hline Calculated stellar $^{\mathrm{a}}$ & $1.6 \times 10^{13}$ \\
\hline
\end{tabular}

Note. ${ }^{\text {a }}$ The total bolometric output at $t=900 \mathrm{Myr}$ (see Figure 3).

So, in principle, all the FIR emission could arise from dust heated by the stellar radiation. In this extreme case, all the observed UV to near-IR luminosity should originate from the AGN. Figure 6 (left panel) depicts the stellar SED calculated for the hierarchical merger SFR at the galactic age of 900 Myr. In the absence of any discriminating line emission, can this extreme case be ruled out on the basis of the 0.1 to $1 \mu \mathrm{m}$ continuum emission? This question is addressed in the right panel of the figure which depicts the probability distribution of photometric spectral indices $\alpha_{p}$ derived from the observed magnitudes of quasars (Richards et al. 2003). The spectral index of the $0.1-1.0 \mu \mathrm{m}$ spectrum of $\mathrm{J} 1148+5251$ is $\alpha_{\nu} \approx-0.35$ (left panel) which corresponds to a value of $\alpha_{p} \approx-1.65$. This value is near the median value of -1.6 . The optical to near-IR spectrum of J1148+5251 therefore has an equally high probability of being entirely that of an AGN as that of a starburst galaxy. The nature of the SED can therefore only be determined with the detection of mid-IR fine structure lines from highly ionized species such as [Ne v]14.3 and $24.3 \mu \mathrm{m}$ which are unambiguous diagnostics of AGN activity (Satyapal et al. 2009; Dudik et al. 2009).

\subsection{The Rarity of Dusty Hyperluminous IR Galaxies at High Redshifts}

The AGB scenario for the formation of dust in J1148+5251 requires the early onset of star formation. Valiante et al. (2009) used the SFR derived in the Li07 hierarchical mergers model as a plausible description of the SFH in J1148+5251. Figure 3 shows that this scenario requires the assembly of galaxies with stellar masses of $M_{\star} \approx 7 \times 10^{11} M_{\odot}$ by $z \approx 9$. The associated mass of the dark matter (DM) halo is: $M_{h} \approx\left(\Omega_{m} / \Omega_{b}\right)\left(M_{\star}+M_{g}\right) \approx$ $4 \times 10^{12} M_{\odot}$. The comoving number density of such halos derived from the Press-Schechter (PS) approximation (Press \& Schechter 1974) is about $10^{-12} \mathrm{Mpc}^{-3}$ (Stiavelli 2009; Li et al. 2007). This number is significantly smaller than the $\sim 10^{-9}$ $\mathrm{Mpc}^{-3}$ comoving volume density of the $z \sim 6$ SDSS QSOs (Fan et al. 2003) which have optical to near-IR luminosities similar to J1148+5251 (Jiang et al. 2010). The apparent three orders of magnitude discrepancy between the observed number density of J1148+5251-like QSOs and that calculated using the PS approximation can probably be attributed to one or more of the following: (1) the breakdown in the accuracy of the formalism at high redshifts and large halo masses ( $\mathrm{Li07}$ and references therein); (2) the observations presented by Jiang et al. (2010) only cover the optical to near-IR wavelengths, which only includes a hot dust component, and thus their QSO sample may therefore not contain a massive amount of colder dust and may therefore not be representative of the population of J1148+5251-type objects at far-IR wavelengths; and (3) the Li07 merger scenario is not the only scenario that can lead to the formation of the supermassive $\mathrm{BH}$, even if the $\mathrm{BH}$-halo mass correlation holds at these redshifts. 

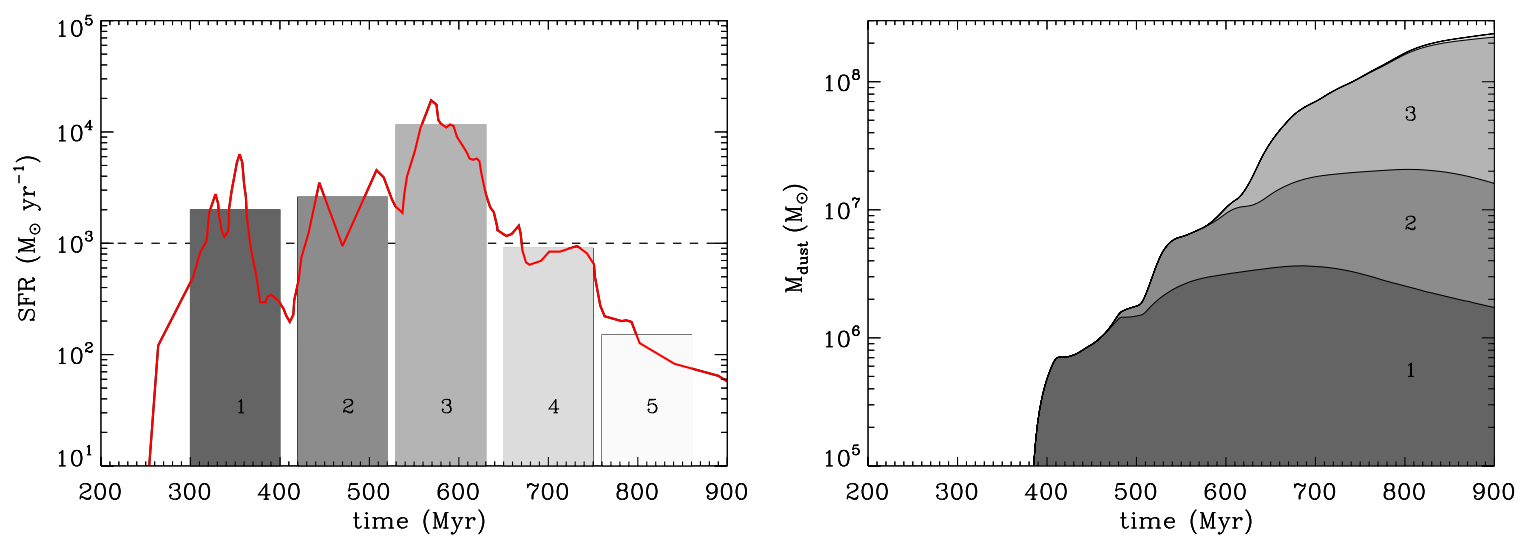

Figure 5. Left panel: the star formation rate of a galaxy going through hierarchical merger episodes (Li07; see also Figure 1 in Li et al. 2008) shown as a bold red line can be approximated by a series of five discrete $100 \mathrm{Myr}$ duration bursts (shaded bars). Right panel: the contribution of each of the five bursts depicted in the left panel to the total dust mass observed at the galactic age of $900 \mathrm{Myr}$. Most of the dust is created by the third burst which had the largest star formation rate and is followed by a period of relatively low rates of star formation and grain destruction. The figure illustrates the sensitivity of the surviving dust mass to the SFH of the galaxy. (A color version of this figure is available in the online journal.)
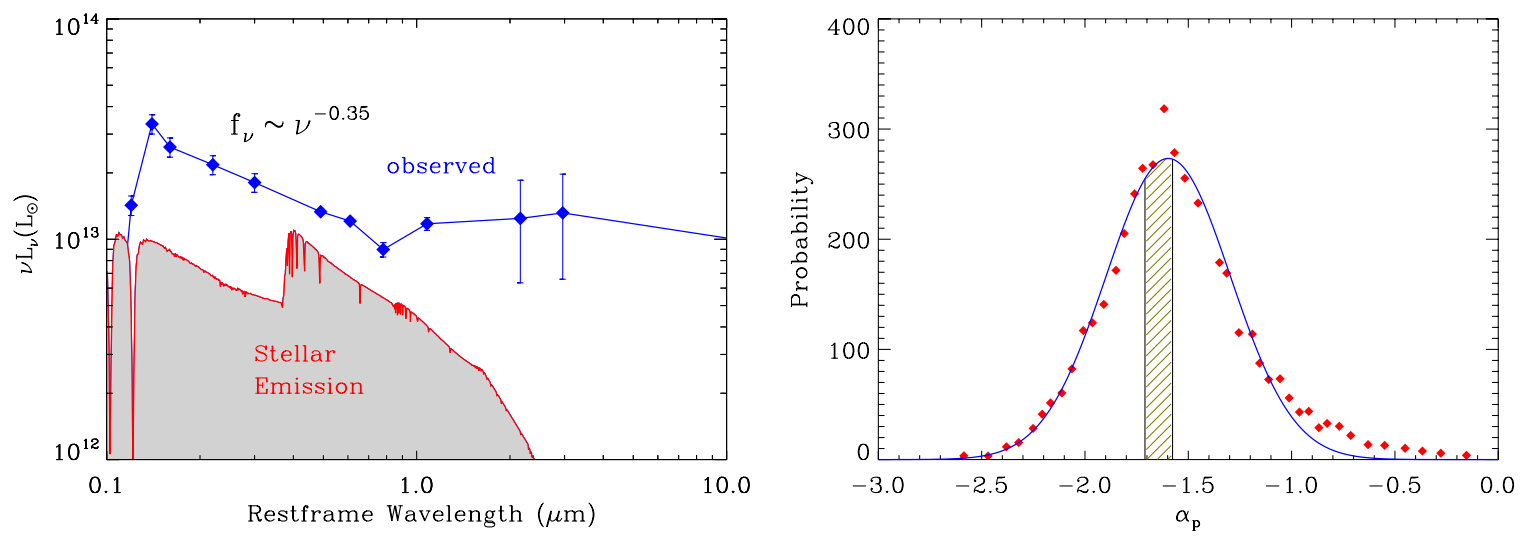

Figure 6. Left panel: comparison of the stellar SED calculated for the hierarchical merger SFR at $z=6.4$ (shaded area) with the observations. In the AGB, most of the optical to near-IR flux is emitted by the AGN. In contrast, in the SN scenario for the origin of the dust, most of this emission originates from stars (see Figure 11 in Dwek et al. 2007). Right panel: the probability distribution of photometric spectral indices $\alpha_{p}$ derived from the observed magnitudes of quasars Richards et al. (2003). The spectral index of $\alpha_{v} \approx-0.35$ (left panel) corresponds to a value of $\alpha_{p} \approx-1.65$. The figure illustrates the fact that there is a high probability that the observed $0.1-1 \mu \mathrm{m}$ flux could be entirely attributed to the emission from an AGN.

(A color version of this figure is available in the online journal.)

In the SN scenario, if we assume that the dust formed in an extremely young burst, the mass of the dark halo associated with an $M_{\star} \approx 10^{11} M_{\odot}$ galaxy is about $6 \times 10^{11} M_{\odot}$. The comoving number density of such halos is about $10^{-5} \mathrm{Mpc}^{-3}$, consistent with the number density of galaxies with similar stellar masses detected in the Great Observatories Origins Deep Survey (GOODS) fields (Stark et al. 2009). If all these galaxies had luminosities similar to J1148+5251, then the comoving bolometric luminosity density at $z \approx 6$ should be about $10^{9} \mathrm{~L}_{\odot} \mathrm{Mpc}^{-3}$. This luminosity density is somewhat larger than the total luminosity density presented in Figure 18 of Franceschini et al. (2010), when extrapolated to $z \approx 6$. However, galaxy count models suggest that galaxies with luminosities above $\sim 2 \times 10^{13} L_{\odot}$ constitute only a small fraction, about $10^{-4}$, of the total number of galaxies at high redshift (Chary \& Elbaz 2001; Franceschini et al. 2010). This gives a comoving number density of J1148+5251-type objects of $\sim 10^{-9} \mathrm{Mpc}^{-3}$, similar to the Fan et al. (2003) observations. So, in the SN scenario a significant fraction of the $z \sim 6$ quasars should have massive amounts of dust. This result is not yet supported by current observations, which show that a significant fraction of these objects are dust-free. However, among the $M_{\star} \approx 10^{11} M_{\odot}$ galaxies, J1148+5251 is an extremely rare object, consistent with the inferred low extinction and metallicities in the $z \sim 7$ galaxies detected in the CDF-South GOODS field with the WFC3/IR camera on the Hubble Space Telescope (Bouwens et al. 2010). The rarity of J1148+5251 in the SN scenario should not be surprising considering the very contrived SFH required to form the inferred mass of dust in this object.

\subsection{The Star Formation Efficiency}

Observations of star-forming galaxies revealed an empirical relation between a galaxy's global SFR and its total gas mass. For a Salpeter IMF, this relation can be written as (Kennicutt 1998b):

$\Sigma_{\mathrm{SFR}}\left(M_{\odot} \mathrm{yr}^{-1} \mathrm{kpc}^{-2}\right)=(2.5 \pm 0.7) \times 10^{-4} \Sigma_{\mathrm{g}}\left(M_{\odot} \mathrm{pc}^{-2}\right)^{1.4 \pm 0.15}$.

Very Large Array (VLA) observations of J1148+5251 show that the $\mathrm{CO}$ gas is distributed over an extended region with an area of $\sim 4 \mathrm{kpc}^{2}$ (Walter et al. 2004). Adopting a total gas mass of $5 \times 10^{10} M_{\odot}$ and assuming that it occupies the same area as the $\mathrm{CO}$ gas gives an expected SFR of $\sim 560 \pm 240 M_{\odot} \mathrm{yr}^{-1}$. For the mass-heavy IMF adopted here, the corresponding SFR 
is $\sim 220 \pm 90 M_{\odot} \mathrm{yr}^{-1}$. This value is larger than the SFR at the epoch of observations in the AGB scenario (see Figure 5, left panel), suggesting that the SF efficiency at that time is about $\sim 3$ times lower than that in normal star-forming galaxies, which varies between $2 \%$ and $10 \%$ (Kennicutt 1998b). However, this instantaneous SF efficiency does not reflect its past value which, averaged over a fixed time interval, should scale as $\Sigma_{g}^{0.4}$. So, the average SF efficiency in $\mathrm{J} 1148+5251$ should be similar to that in normal SF galaxies.

Studies of the relation between the mass of DM halos and the luminosity of their host galaxies show an evolutionary trend between the galaxies' luminosity function and their halo masses (Trenti et al. 2010). This trend can be translated into a relation between their SF efficiency, $\eta$, and their halo mass. For masses above $10^{11} M_{\odot}$, the SF efficiency depends only mildly on halo mass and is given by $\eta \approx 0.06\left(M_{h} / 10^{11} M_{\odot}\right)^{0.3}$ (Trenti et al. 2010). For the inferred halo mass of $4 \times 10^{12} M_{\odot}$ in the AGB scenario, $\eta \approx 0.18$. So, the predicted SF efficiency is somewhat higher than the inferred average in the AGB scenario, which can however be attributed to expected large variations in individual objects.

In the $\mathrm{SN}$ scenario, all the far-IR luminosity is powered by star formation, suggesting a SFR in excess of $\sim 3000 M_{\odot} \mathrm{yr}^{-1}$. This implies a very high SF efficiency for the given mass of the galaxy. At this rate, the galaxy will deplete its reservoir of gas in about $10 \mathrm{Myr}$, suggesting that we are just witnessing the onset of a large burst of SF in this object, which will be rapidly quenched by stellar feedback.

\section{ALTERNATIVE DUST SOURCES}

The still unresolved discrepancy between the predicted and inferred stellar masses in the AGB scenario and the specialized SF scenario required to form the inferred J1148+5251 dust mass by $\mathrm{SNe}$, raises the need to explore alternative, non-stellar sources that may produce the large amount of dust in this object. In the following, we consider the growth of dust in MCs and their formation around AGNs as such sources.

\subsection{Molecular Clouds}

Numerous observations and theoretical considerations support the notion that dust grains are processed and grow in dense MCs. Observationally, extinction measurements show that the value of $R_{V} \equiv A_{V} / E(B-V)$, an indicator of grain size, increases with hydrogen column density (e.g., Kandori et al. 2003 and references therein). Similarly, interstellar depletions of refractory elements increase with average gas column density (Savage \& Sembach 1996; Jenkins 2009) and infrared spectroscopy of MCs show the presence of ices, expected to have condensed out on interstellar grain cores (van Dishoeck 2004). Theoretically, the discrepancy between the relatively short lifetime of interstellar dust (Jones 2004) and the longer lifetime for their replenishment by $\mathrm{SNe}$ or AGB stars has been attributed to grain growth in MCs (Dwek \& Scalo 1980; Dwek 1998; Tielens 1998; Zhukovska et al. 2008). In particular, the possibility that rapid growth of dust in high-redshift galaxies took place in MCs was considered by Michałowski et al. (2010). Here, we provide a more quantitative discussion of such possibility.

Several conditions must be met for grain growth in clouds to be significant: (1) the presence of preexisting refractory grain cores onto which the accretion can take place and (2) the accretion time onto these cores must be shorter than the lifetime of the dense clouds in which this process is taking place.
Let $\rho_{g r}=n_{g r} \times m_{g r}$ be the mass density of grains in the MC, where $n_{g r}$ is their number density and $m_{g r}$ their mass. Assuming that all grains have the same radius $a$ and density $\rho$, the growth rate of $\rho_{g r}$ due to accretion of a heavy element $A$ with a molecular weight $\mu_{A}$ and number density $n_{A}$ is given by

$$
\frac{d \rho_{g r}}{d t}=\alpha \pi a^{2} \mu_{A} n_{A} n_{g r} \widetilde{v}
$$

where $\alpha$ is the sticking coefficient in the collision, and $\widetilde{v}=\left(8 k T / \pi \mu_{A}\right)^{1 / 2}$ is the mean thermal velocity of $A$ at temperature $T$.

The accretion time, $\tau_{\mathrm{acc}}$, is defined as follows:

$$
\begin{aligned}
\tau_{\mathrm{acc}}^{-1} \equiv \frac{1}{\rho_{g r}} \frac{d \rho_{g r}}{d t} & =\alpha\left(\frac{\pi a^{2}}{m_{g r}}\right) \mu_{A} n_{A} \widetilde{v} \\
& =\alpha\left(\frac{3}{4 \rho a}\right)\left(\frac{\mu_{A}}{m_{\mathrm{H}}}\right)\left(\frac{n_{A}}{n_{\mathrm{H}_{2}}}\right) m_{\mathrm{H}} n_{\mathrm{H}_{2}} \widetilde{v} .
\end{aligned}
$$

Taking the mass of an oxygen atom to represent that of a colliding species, so that $\mu_{A} / m_{\mathrm{H}}=16$, and $n_{A} / n_{\mathrm{H}_{2}} \approx 5 \times 10^{-4}$ (assuming solar abundances), we get

$$
\tau_{\mathrm{acc}}(\mathrm{s})=\frac{2 \times 10^{18}}{\alpha} \rho\left(\frac{\mathrm{a}}{\mu \mathrm{m}}\right)\left(\frac{\mathrm{n}_{\mathrm{H}_{2}}}{\mathrm{~cm}^{-3}}\right)^{-1}\left(\frac{\mathrm{T}}{\mathrm{K}}\right)^{-1 / 2} .
$$

For a grain density of $3 \mathrm{~g} \mathrm{~cm}^{-3}$, radius of $0.1 \mu \mathrm{m}$, a cloud density of $10^{3} \mathrm{~cm}^{-3}$, and cloud temperature of $20 \mathrm{~K}$, the accretion time becomes $\tau_{\text {acc }} \sim 3 \times 10^{6} \mathrm{yr}$.

The lifetime, $\tau_{c}$, of the MCs is given by

$$
\tau_{c}=\frac{M_{c}}{\psi} \approx \frac{2 \times 10^{10}}{3000} \approx 7 \times 10^{6} \mathrm{yr}
$$

where $M_{c}$ is the MC mass and where we used the inferred $\mathrm{H}_{2}$ mass and SFR to calculate the average lifetime of the molecular gas. We used here the large SFR inferred from the SN scenario to minimize the lifetime of the MC. Had we used the SFR from Figure 5, the MC lifetime would have been higher by a factor of $\sim 50$. Within the uncertainties of the chosen values of the various parameters, the cloud lifetime is not significantly shorter than the accretion time. Consequently, grain growth in the dense ISM can play an important role in determining the observed dust mass.

The accreted material may be in the form of organic refractory material (Greenberg et al. 1995). The main observational constraints, extinction, diffuse IR emission, and interstellar abundances in the MW can also be met with composite dust models that incorporate such material (Li \& Greenberg 1997; Zubko et al. 2004). If so, then the dust of $\mathrm{J} 1148+5251$ could also consist of such composite grains that could have a significant abundance in the diffuse ISM. In the MW, most $(60 \%-75 \%)$ of the IR emission is radiated by dust in the diffuse ISM, with only 15\%-30\% radiated from MCs (Sodroski et al. 1997). Likewise, the IR emission from $\mathrm{J} 1148+5251$ could arise either from the dust in the MCs, or from dust in the diffuse ISM, including H II regions. The origin of the IR emission in this case can therefore only be determined by considering dust evolution models that incorporate a two-phase medium and take the physical processes and the cycling between the phases into account. 


\section{2. "Smoking Quasars"}

The concept of "smoking quasars," that is, the possibility that quasars may be producers of interstellar dust was first put forward by Elvis et al. (2002). In his scenario, the broad emission line clouds (BELCs), confined by the centrifugally driven outflowing wind (Kartje \& Königl 1996), go through the same density and temperature conditions that characterize the dusty winds in stellar outflows. It is therefore natural to postulate that if the conditions in the stellar winds are ripe to form dust, a similar process will be taking place in the BELCs. This idea was further discussed by Maiolino et al. (2006).

The main issue, not addressed in these papers, is whether the BELCs were dusty to start with (Elitzur \& Shlosman 2006). If so, then the IR emission observed in the vicinity of the AGN is not the signature of radiation from newly formed dust, but that of reprocessed dust. This dust could have formed previously in $\mathrm{SNe}$ or dusty stellar winds. If so, quasars cannot be considered as net producers of interstellar dust. The issue of quasars as dust sources can therefore only be settled once we have a clearer understanding the origin of the BELCs and of the dust formation process in these objects.

\section{DISCUSSION AND SUMMARY}

Using our new integral solutions for the chemical evolution of the ISM gas and its metal and dust contents, we examined the origin of the large amount of dust discovered in the high- $z$ quasar $\mathrm{J} 1148+5251$. We have confirmed that AGB stars can produce the required amount of dust in a merger scenario in which the assembled galaxy undergoes a period of intense star formation at $z \approx 9$, followed by a period of significantly lower stellar activity. In this AGB scenario, an average $20 M_{\odot}$ SN needs to make at least $\sim 1 M_{\odot}$ of dust in order to be a viable source of dust at this high redshift. However, $\mathrm{SNe}$ can yet produce the required amount of dust with a significantly lower dust yield, provided the galaxy underwent a very special SFH. In the following, we summarize the main results of our paper, and discuss future observations and studies needed to distinguish between the two scenarios in order to determine the origin and nature of the dust in J1148+5251 and similar objects in the early universe.

1. The AGB scenario. A galaxy must be at least 400 Myr old for AGB stars to have made a significant contribution to its reservoir of dust, a conditioned fulfilled by the merger scenario for the formation of $\mathrm{J} 1148+5251$. However, this $\mathrm{SFH}$ is not unique, and we outlined several alternative scenarios for the formation of large amounts of dust with AGB stars. At any given epoch, the intensity of the starburst must be at least $2000 M_{\odot} \mathrm{yr}^{-1}$ in order to produce the inferred amount of dust, and the SFR must have dropped to less than $\sim 50 M_{\odot} \mathrm{yr}^{-1}$ thereafter so that the dust that is injected at the epoch of observations will not be significantly destroyed.

2. The $\mathrm{SN}$ scenario. In general, an average $\mathrm{SN}$ must condense about $1 M_{\odot}$ of dust in order to produce the observed amount of dust in J1148+5251. Our current state of knowledge, observationally and theoretically, suggests that the explosive ejecta of a progenitor $\sim 20 M_{\odot}$ star produces only about $0.1-0.15 M_{\odot}$ of dust. Such progenitors are the most common metal-producing $\mathrm{SNe}$ and fall short of meeting the production requirement. However, our knowledge is based on a limited number of observations and calculations, so it may change in the future. Similarly to the alternative AGB scenario, $\mathrm{SNe}$ can produce the observed amount of dust with a dust yield of $\sim 0.15 M_{\odot}$ in a single short-duration and intense burst of star formation, followed by a period of very low star-forming activity. Such scenarios must be very rare in order to explain the low comoving number density of hyperluminous IR objects at high redshifts.

3. The stellar mass and gas content of $\mathbf{J 1 1 4 8 + 5 2 5 1}$. The stellar mass offers an important integral constraint on the duration and intensity of the burst of star formation. These place integral constraints on the total amount of dust that could have formed in the quasar. In the $\mathrm{SN}$ scenario, $M_{\star} \approx 10^{11} M_{\odot}$ of stars are formed around $z \approx 7$. So if dust formation was very efficient in these objects, the SN scenario would produce more dusty hyperluminous IR stars than currently observed at high redshifts. The formation of SN dust must therefore be a very rare event in these objects, limited by the dust formation and grain destruction efficiencies in these objects. The AGB scenario requires the formation of $M_{\star} \approx 7 \times 10^{11} M_{\odot}$ at redshifts of $\sim 8-9$, suggesting that $\mathrm{J} 1148+5251$ is a very rare event, but perhaps consistent with the number of J1148+5251like QSOs detected at $z \sim 6$. If so, a significant fraction of $z \sim 6$ quasars should have massive amounts of dust, a prediction that is yet unsupported by current observations. Furthermore, the stellar mass produced in the AGB scenario is much larger that the inferred dynamical mass of the galaxy. Resolving this discrepancy is a crucial step in determining the origin of dust in $\mathrm{J} 1148+5251$.

4. The nature of the optical to near-IR SED of J1148+5251. In the SN scenario, all the emission in this wavelength region is produced by stars, whereas in the AGB scenario all this emission is produced by the AGN. In the absence of a detailed spectrum, we used the slope of the SED to discriminate between the two scenarios. The results show that the slope of the SED is typical of quasars and also consistent with that produced by an extincted burst of star formation. The detection of mid-IR fine structure lines that serve as unambiguous diagnostics of AGN or starburst activity will be extremely useful for distinguishing between the two scenarios.

5. The efficiency of grain destruction in the ISM. Our current state of knowledge is predominantly based on the simulations that were performed for a homogeneous ISM. These calculations need to be expanded for a two-phase ISM, taking also the spatial distribution of dust sources and sinks into account. For example, one can easily envision an intense starburst or spatially isolated $\mathrm{SNe}$ producing a large amount of dust in one location in the galaxy, followed sometime later by another physically isolated starburst that will have little effect on the destruction of the dust created in the first. Spatially isolated starbursts or SNe may therefore increase the currently estimated short lifetime of the dust in the ISM.

6. Dust growth in the ISM. Accretion in MCs must play an important role in explaining the origin of the Galactic pattern of interstellar depletions. A necessary condition for this process to be important is that the lifetime of MCs must be larger than the timescale for grain growth by accretion. This condition is met in $\mathrm{J} 1148+5251$, rendering it a possible, but not necessary, source for the growth of dust in this object. Detailed information of the correlation of the IR emission with the different gas phases in J1148+5251 may resolve this ambiguity. 
7. Dust formation in and around the AGN. The effectiveness of AGNs as sources of newly formed dust remains unclear. The origin of the dense broad-line-emitting clouds, and whether they were initially dusty so that the AGN merely reformed pre-existing dust, are questions that need to be addressed to ascertain their role as dust sources in quasars.

E.D. thanks the Department of Astronomy at the University of Maryland and the Department of Astronomy and Astrophysics at Tel Aviv University for their hospitality during the time of the writing of this paper. E.D. also acknowledges helpful discussions with Richard Mushotzky, Sylvain Veilleux, Amiel Sternberg, Dan Laor, and Hagai Netzer, and thanks Rick Arendt and Dieter Hartmann for comments on parts of the manuscript. E.D. also thanks Massimo Stiavelli for providing an expanded version of Figure 2.6 in his book for use in this study. Finally, we thank the anonymous referee for useful comments that have led to significant improvements in the paper.

\section{REFERENCES}

Audouze, J., \& Tinsley, B. M. 1976, ARA\&A, 14, 43

Barlow, M. J., et al. 2010, A\&A, 518, L138

Bouwens, R. J., et al. 2010, ApJ, 708, L69

Bromm, V., \& Larson, R. B. 2004, ARA\&A, 42, 79

Chary, R., \& Elbaz, D. 2001, ApJ, 556, 562

Cherchneff, I., \& Dwek, E. 2010, ApJ, 713, 1

Crowther, P. A. 1997, MNRAS, 290, L59

Diehl, R., et al. 2006, Nature, 439, 45

Donn, B., \& Nuth, J. A. 1985, ApJ, 288, 187

Dudik, R. P., Satyapal, S., \& Marcu, D. 2009, ApJ, 691, 1501

Dunne, L., Eales, S., Ivison, R., Morgan, H., \& Edmunds, M. 2003, Nature, 424, 285

Dunne, L., et al. 2009, MNRAS, 394, 1307

Dwek, E. 1998, ApJ, 501, 643

Dwek, E. 2005, in AIP Conf. Ser. 761, The Spectral Energy Distributions of Gas-Rich Galaxies: Confronting Models with Data, ed. C. C. Popescu \& R. J. Tuffs (Melville, NY: AIP), 103

Dwek, E., Galliano, F., \& Jones, A. P. 2007, ApJ, 662, 927

Dwek, E., \& Scalo, J. M. 1980, ApJ, 239, 193

Elitzur, M., \& Shlosman, I. 2006, ApJ, 648, L101

Elvis, M., Marengo, M., \& Karovska, M. 2002, ApJ, 567, L107

Fan, X., et al. 2003, AJ, 125, 1649

Ferrarotti, A. S., \& Gail, H.-P. 2006, A\&A, 447, 553

Fioc, M., \& Rocca-Volmerange, B. 1997, A\&A, 326, 950

Franceschini, A., Rodighiero, G., Vaccari, M., Berta, S., Marchetti, L., \& Mainetti, G. 2010, A\&A, 517, A74

Galliano, F., Dwek, E., \& Chanial, P. 2008, ApJ, 672, 214

Greenberg, J. M., \& Li, A. 1999, Adv. Space Res., 24, 497

Greenberg, J. M., et al. 1995, ApJ, 455, L177
Greggio, L. 2005, A\&A, 441, 1055

Greggio, L. 2010, MNRAS, 406, 22

Harries, T. J., Monnier, J. D., Symington, N. H., \& Kurosawa, R. 2004, MNRAS, 350,565

Jenkins, E. B. 2009, ApJ, 700, 1299

Jiang, L., et al. 2010, Nature, 464, 380

Jones, A. P. 2004, in ASP Conf. Ser. 309, Astrophysics of Dust, ed. A. N. Witt, G. C. Clayton, \& B. T. Draine (San Francisco, CA: ASP), 347

Kandori, R., Dobashi, K., Uehara, H., Sato, F., \& Yanagisawa, K. 2003, AJ, 126, 1888

Karakas, A., \& Lattanzio, J. C. 2007, PASA, 24, 103

Kartje, J. F., \& Königl, A. 1996, Vistas Astron., 40, 133

Kennicutt, Jr., R. C. 1998a, ARA\&A, 36, 189

Kennicutt, Jr., R. C. 1998b, ApJ, 498, 541

Kormendy, J., \& Gebhardt, K. 2001, in AIP Conf. Ser. 586, 20th Texas Symposium on Relativistic Astrophysics, ed. J. C. Wheeler \& H. Martel (Meleville, NY: AIP), 363

Li, A., \& Greenberg, J. M. 1997, A\&A, 323, 566

Li, Y., et al. 2007, ApJ, 665, 187

Li, Y., et al. 2008, ApJ, 678, 41

Liffman, K., \& Clayton, D. D. 1989, ApJ, 340, 853

Maiolino, R., et al. 2004, Nature, 431, 533

Maiolino, R., et al. 2006, Mem. Soc. Astron. Ital., 77, 643

Michałowski, M. J., Murphy, E. J., Hjorth, J., Watson, D., Gall, C., \& Dunlop, J. S. 2010, A\&A, 522, A15

Morgan, H. L., \& Edmunds, M. G. 2003, MNRAS, 343, 427

Nozawa, T., Kozasa, T., Umeda, H., Maeda, K., \& Nomoto, K. 2003, ApJ, 598, 785

Nozawa, T., et al. 2010, ApJ, 713, 356

Prantzos, N., Doom, C., De Loore, C., \& Arnould, M. 1986, ApJ, 304, 695

Press, W. H., \& Schechter, P. 1974, ApJ, 193, 437

Raiteri, C. M., Villata, M., \& Navarro, J. F. 1996, A\&A, 315, 105

Rho, J., et al. 2008, ApJ, 673, 271

Richards, G. T., et al. 2003, AJ, 126, 1131

Satyapal, S., et al. 2009, ApJ, 704, 439

Savage, B. D., \& Sembach, K. R. 1996, ARA\&A, 34, 279

Shields, G. A., Menezes, K. L., Massart, C. A., \& Vanden Bout, P. 2006, ApJ, 641,683

Sibthorpe, B., et al. 2010, ApJ, 719, 1553

Snow, Jr., T. P. 1975, ApJ, 202, L87

Sodroski, T. J., et al. 1997, ApJ, 480, 173

Spergel, D. N., et al. 2007, ApJS, 170, 377

Stark, D. P., et al. 2009, ApJ, 697, 1493

Stiavelli, M. 2009, From First Light to Reionization: The End of the Dark Ages (Weinheim: Wiley-VCH)

Tielens, A. G. G. M. 1998, ApJ, 499, 267

Trenti, M., et al. 2010, ApJ, 714, L202

Valiante, R., Schneider, R., Bianchi, S., \& Andersen, A. C. 2009, MNRAS, 397, 1661

van Dishoeck, E. F. 2004, ARA\&A, 42, 119

Walter, F., et al. 2004, ApJ, 615, L17

Willott, C. J., McLure, R. J., \& Jarvis, M. J. 2003, ApJ, 587, L15

Zhukovska, S., Gail, H., \& Trieloff, M. 2008, A\&A, 479, 453

Zubko, V., Dwek, E., \& Arendt, R. G. 2004, ApJS, 152, 211 